\title{
Are Movement Preparation and Movement Initiation Truly Independent?
}

\author{
Isobel Weinberg \\ Sobell Department of Motor Neuroscience and Movement Disorders, UCL Institute of Neurology, University College London, London WC1N 3BG, United \\ Kingdom \\ Review of Haith et al.
}

The use of reaction times in cognitive neuroscience research is pervasive; reaction time distributions are used to make inferences about the neural processes that underlie them. A central assumption in these inferences is that reaction times signify the cumulative time needed to produce a movement. Yet there are intriguing hints that humans can produce accurate movements with a markedly shorter latency than a standard reaction time. For instance, when startled by a loud auditory stimulus, humans initiate a movement with half the normal reaction time (VallsSolé et al., 1999). Crucially, this is a specific movement prepared in response to a stimulus and not a generalized startle response. Relatedly, neural conduction delays account for just $30 \%$ of reaction time (Carpenter, 1999). In a recent paper in The Journal of Neuroscience, Haith et al. (2016) set out to systematically investigate the assertion that successful movements can be initiated in less time

Received April 5, 2016; revised May 20, 2016; accepted May 20, 2016.

This work was supported by a University College London MBPhD studentship, funded by UCL Hospitals Biomedical Research Centre. I thank Archy de Berker and Sven Bestmann for helpful discussion and comments on an earlier version of the paper.

The authors declare no competing financial interests.

Correspondence should be addressed to Isobel Weinberg, Sobell Department of Motor Neuroscience and Movement Disorders, UCL Institute of Neurology, University College London, London WC1N 3BG, UK. E-mail: Isobel.weinberg.13@ucl.ac.uk.

DOI:10.1523/JNEUROSCI.1135-16.2016

Copyright $\odot 2016$ the authors $\quad 0270-6474 / 16 / 367076-03 \$ 15.00 / 0$ than suggested by the voluntary reaction time.

In their "Forced RT" paradigm, Haith et al. (2016) required human subjects to move at a fixed initiation cue. Before the cue, subjects saw a target that indicated which direction they should reach. Critically, participants had to move when the initiation cue appeared, regardless of whether there had been enough time after target appearance to prepare a movement in the correct direction. The experimenters shifted the time of target appearance relative to that of the initiation cue to find out how long subjects needed to make an accurate movement. As anticipated, they found that subjects could produce an accurate movement with $\sim 80$ ms less preparation time than their normal reaction time. Haith et al. (2016) used the accuracy at various target-cue intervals to infer the underlying distribution of this "motor preparation time." The authors reasoned that if preparation of an accurate movement is completed in $\sim 100 \mathrm{~ms}$ less than a voluntary reaction time, an alternative signal must determine the time taken to start moving, and they call this "motor initiation."

What is the relationship between motor preparation and motor initiation? To answer this question, Haith et al. (2016) analyzed their data for errors in target choice, and found that subjects made more errors when forced reaction times were shorter. They argue that this is incompatible with motor preparation and initiation being yoked (with motor initiation being triggered by the completion of motor preparation), because this would lead to errors being distributed evenly across reaction times. Instead, they propose that errors occur on trials in which motor initiation time elapses before movement preparation is complete. In support of this, they use the inferred distributions of movement planning time to predict error rates in each subject and show a good correspondence.

What if the improvement in reaction times compared with baseline was caused by insufficient motivation when baseline reaction times were measured? To address this concern, Haith et al. (2016) designed a second experiment, identical to the baseline condition, except that a shrinking cue indicated a strict deadline to the participants. This deadline was initially 900 $\mathrm{ms}$ and became progressively more stringent over the course of the experiment. This stressful condition led to a reaction time improvement of only $\sim 8 \mathrm{~ms}$ (compared to $80 \mathrm{~ms}$ improvement previously). Beyond this, further speed improvements came at an accuracy cost.

The authors suggest that their results herald "a significant departure from the traditional view of the RT (reaction time) as the total summed duration of component processes that must occur before movement." Others have previously challenged this conceptualization of reaction times (Cisek, 2007) and this paper now uses a simple behavioral par- 


\section{A Urgency-gating model (Cisek et al, 2009):}

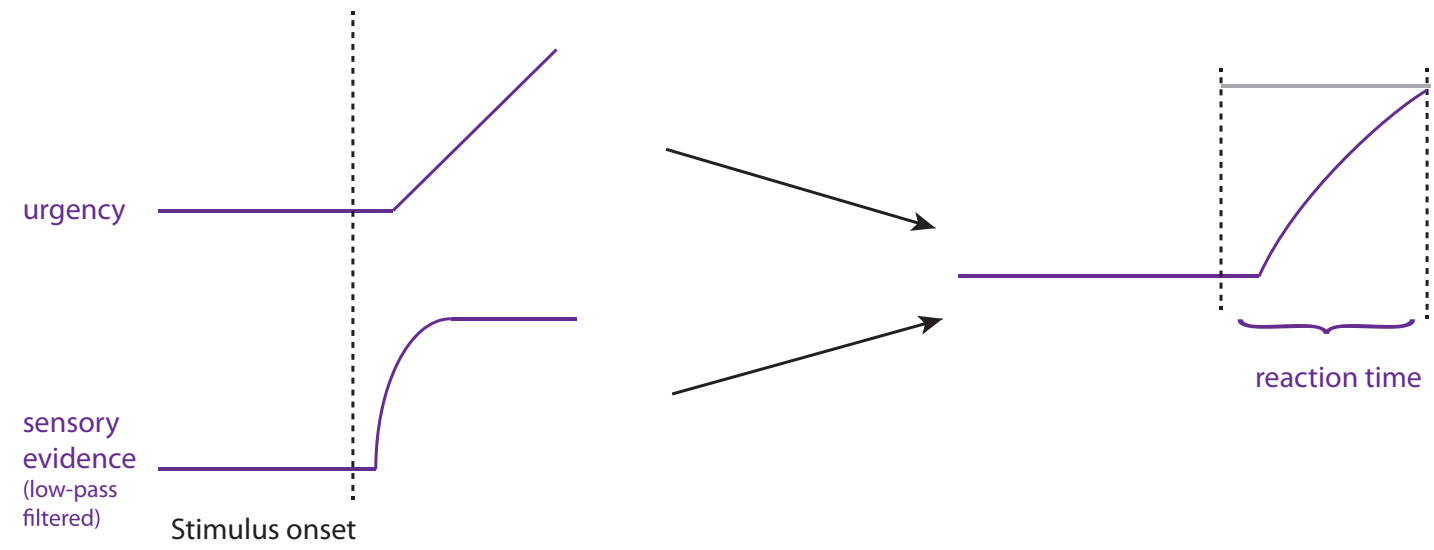

\section{B Motor initiation model (Haith et al, 2016):}
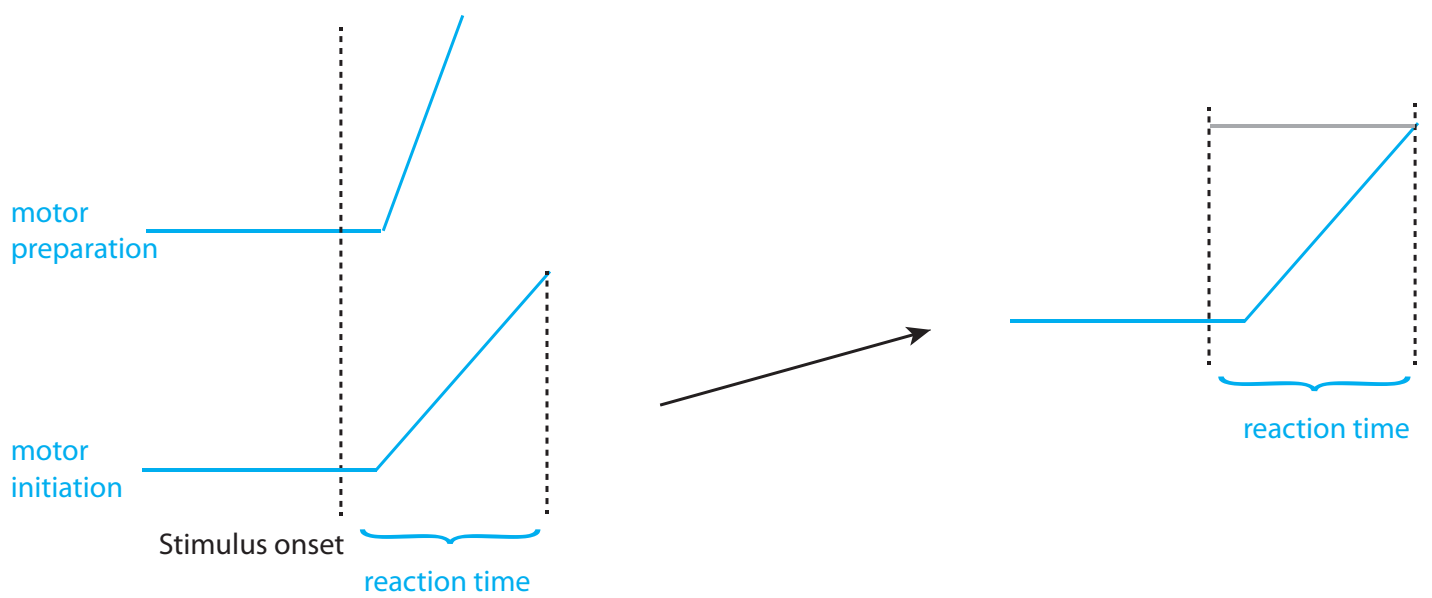

Figure 1. In the urgency-gating model, reaction time is a function of a rising urgency signal and low-pass filtered sensory evidence [A; adapted with permission from Cisek et al. (2009)]. By contrast, in Haith et al.'s (2016) paper, reaction time is determined solely by the time taken for motor initiation (B).

adigm (Ghez et al., 1997; Stanford et al., 2010) to isolate the timing of underlying processes. The paradigm allows one to divorce movement preparation time from reaction time. This opens up the possibility of investigating factors that modulate movement preparation time behaviorally. For instance, a follow-up experiment might ask whether movement preparation time increases with increasing complexity of the planned movement.

In Haith et al.'s (2016) interpretation, motor initiation time determines reaction times. This is a departure from widely accepted models in which the time taken for the decision about where to move is the critical determinant of reaction times (Gold and Shadlen, 2007). A recent challenger to these established models is Cisek's urgency-gating model (Cisek et al., 2009), to which Haith et al.'s (2016) interpretation has compelling parallels. In the urgency-gating model, a growing "urgency" signal drives the rise to threshold behavior characteristically seen in parietal and frontal neuronal responses during a decision (eg, Roitman and Shadlen, 2002). Thus, the mounting neuronal activity is driven not by temporal integration of sensory evidence, as is widely accepted, but by a steadily rising urgency signal that is combined with a momentary estimate of sensory information. While the urgency-gating model was formulated to explain single-neuron responses, and so is different in scope from Haith et al.'s (2016) data, both share the key feature that the signal that determines reaction times represents intention to move independently of movement planning itself. Evidence for Cisek's model has to date come from modeling reaction time distributions and neuronal recordings (Cisek et al., 2009; Thura and Cisek, 2014). By attempting to dissociate an urgency/initiation signal behaviorally, Haith et al.'s (2016) paper provides evidence from a different angle.

The theoretical appeal of dissociating action initiation from decision/action preparation is that it offers a mechanism for self-timed movements (Brass and Haggard, 2008). Although cognitive neuroscience discussions often focus on modeling the process of responding to a laboratory stimulus, the inclusion of a mechanism for determining timing, as well as response, bring us closer to a naturalistic model is which movements are self-timed as well as self-chosen. Indeed, as Cisek et al. (2009) points out, there is evidence for this viewpoint in macaque parietal neuronal recordings from Roitman and Shadlen (2002). Before a selfpaced movement, neural activity shows a 
steady rise, but in a version where the monkey moves at a fixed time, ramping activity is not seen in the neuron until the end of the decision, consistent with this ramp being driven by a motor initiation/ urgency signal that appears only when it is time to make the movement.

Although a distinct motor initiation signal may be theoretically appealing, there are fundamental features of reaction times that any model must attempt to explain. First, behavior shows an established tradeoff between speed and accuracy. The mechanism for this in Haith et al.'s (2016) model is that, in faster decisions, it is more likely that movement initiation time has elapsed before motor preparation is complete. However, this does not explain a second ubiquitous finding in reaction time experiments: more difficult decisions take longer. Evidence accumulator models intuitively demonstrate this behavior: weak evidence takes longer to accumulate to a threshold. How would the Haith et al. (2016) conceptualization produce this behavior, and avoid movements based on tricky evidence being released too early and thus incorrectly? For movement initiation time to reflect choice difficulty, a linkage between motor preparation and initiation seems necessary, but Haith et al.'s (2016) analyses exclude a simple temporal dependence. The authors suggest that movement initiation is delayed relative to mean movement preparation time, but this could not offer a flexible mechanism for adjusting movement initiation time on a movement-tomovement basis.
To account for this behavioral finding, I propose a slight shift in interpretation. In Cisek et al.'s (2009) model, neural activity is a combination of evidence and urgency and the two together determine reaction time (Fig. 1A). This is fundamentally different from Haith et al.'s (2016) model, in which reaction time is determined by movement initiation time alone (Fig. $1 B$ ). We could instead imagine a reinterpretation of their results in which, similarly to in Cisek et al.'s (2009) neuronal model, the movement initiation signal combines with motor preparatory activity and the level of their combined activity is the ultimate determinant of reaction time. Do the core findings of the paper still stand under this scheme? Yes: this does not change the explanatory power of an initiation signal for understanding the speeded reaction times. However, combining this signal with an action preparation signal offers a mechanism for more difficult decisions taking longer: movement preparation builds up more slowly. Indeed, Haith et al. [(2016), their Fig. 2E] plot movement time against initiation time, and the two appear to be correlated across individuals, which is in keeping with a role for motor preparation in determining reaction times alongside motor initiation.

In short, Haith et al.'s (2016) paper offers an inventive method for separating the time taken to plan an accurate movement from the reaction time, and thus studying their determinants independently. It illustrates how elegantly designed behavioral experiments continue to have power to address fundamental questions.

\section{References}

Brass M, Haggard P (2008) The what, when, whether model of intentional action. Neuroscientist 14:319-325. CrossRef Medline

Carpenter RH (1999) A neural mechanism that randomises behaviour. J Conscious Stud 6:13-22.

Cisek P (2007) Cortical mechanisms of action selection: the affordance competition hypothesis. Philos Trans R Soc Lond B Biol Sci 362: 1585-1599. CrossRef Medline

Cisek P, Puskas GA, El-Murr S (2009) Decisions in changing conditions: the urgency-gating model. J Neurosci 29:11560-11571. CrossRef Medline

Ghez C, Favilla M, Ghilardi MF, Gordon J, Bermejo R, Pullman S (1997) Discrete and continuous planning of hand movements and isometric force trajectories. Exp Brain Res 115:217-233. CrossRef Medline

Gold JI, Shadlen MN (2007) The neural basis of decision making. Annu Rev Neurosci 30: 535-574. CrossRef Medline

Haith AM, Pakpoor J, Krakauer JW (2016) Independence of movement preparation and movement initiation. J Neurosci 36:30073015. CrossRef Medline

Roitman JD, Shadlen MN (2002) Response of neurons in the lateral intraparietal area during a combined visual discrimination reaction time task. J Neurosci 22:9475-9489. Medline

Stanford TR, Shankar S, Massoglia DP, Costello MG, Salinas E (2010) Perceptual decision making in less than 30 milliseconds. Nat Neurosci 13:379-385. CrossRef Medline

Thura D, Cisek P (2014) Deliberation and commitment in the premotor and primary motor cortex during dynamic decision making. Neuron 81:1401-1416. CrossRef Medline

Valls-Solé J, Rothwell JC, Goulart F, Cossu G, Muñoz E (1999) Patterned ballistic movements triggered by a startle in healthy humans. J Physiol 516:931-938. CrossRef Medline 